\title{
INTRAVESICAL ELECTROMOTIVE MITOMYCIN C VERSUS PASSIVE TRANSPORT MITOMYCIN C FOR HIGH RISK SUPERFICIAL BLADDER CANCER: A PROSPECTIVE RANDOMIZED STUDY
}

\author{
SAVINO M. DI STASI,* ANTONELLA GIANNANTONI, ROBERT L. STEPHEN, $\dagger$ GIOVANNI CAPELLI, \\ PIERLUIGI NAVARRA, RENATO MASSOUD AND GIUSEPPE VESPASIANI
}

From the Departments of Urology and Clinical Biochemistry, Tor Vergata University and Institute of Pharmacology, Catholic University, Rome, Department of Urology, University of Perugia, Perugia, Physion Laboratories, Medolla and Department of Science and Society, University of Cassino, Cassino, Italy

\section{ABSTRACT}

Purpose: In laboratory studies electromotive mitomycin C (MMC) demonstrated markedly increased transport rates compared with passive transport. We performed a prospective study in patients with high risk superficial bladder cancer to assess the efficacy of intravesical electromotive vs passive MMC using bacillus Calmette-Guerin (BCG) as a comparative treatment.

Materials and Methods: Following transurethral resection and multiple biopsies 108 patients with multifocal Tis, including 98 with T1 tumors, were randomized into 3 equal groups of 36 each who underwent $40 \mathrm{mg}$ electromotive MMC instillation with $20 \mathrm{~mA}$ electric current for 30 minutes, $40 \mathrm{mg}$ passive MMC with a dwell time of 60 minutes or $81 \mathrm{mg}$ BCG with a dwell time of 120 minutes. Patients were scheduled for an initial 6 weekly treatments, a further 6 weekly treatments for nonresponders and a followup 10 monthly treatments for responders. Primary end points were the complete response rate at 3 and 6 months. MMC pharmacokinetics were assessed.

Results: The complete response for electromotive vs passive MMC at 3 and 6 months was $53 \%$ versus $28 \%(\mathrm{p}=0.036)$ and $58 \%$ versus $31 \%(\mathrm{p}=0.012)$. For $\mathrm{BCG}$ the responses were $56 \%$ and $64 \%$. Median time to recurrence was 35 vs 19.5 months $(\mathrm{p}=0.013)$ and for BCG it was 26 months. Peak plasma MMC was significantly higher following electromotive MMC than after MMC ((43 vs $8 \mathrm{ng} / \mathrm{ml}$ ), consistent with bladder content absorption.

Conclusions: Intravesical electromotive administration increases bladder uptake of MMC, resulting in an improved response rate in cases of high risk superficial bladder cancer.

KEY WoRDs: bladder, bladder neoplasms, chemotherapy, electricity, mitomycin

Approximately $75 \%$ of patients with bladder cancer present with superficial disease. Only $2 \%$ to $4 \%$ of simple, low grade stage Ta cancers progress, while stage $\mathrm{T} 1$ is more threatening with $20 \%$ to $30 \%$ likely to progress. $^{1}$ Higher disease grades enhance progression, ${ }^{2}$ as does associated Tis. ${ }^{3}$ Intravesical anticancer therapy is appropriate treatment for high risk superficial bladder cancer even if the ultimate long-term benefits are in doubt. ${ }^{4}$ Investigators have described superior results with intravesical bacillus CalmetteGuerin (BCG) compared with chemotherapeutic drugs and they also attributed more numerous and more severe side effects to BCG. ${ }^{5}$

A chemotherapeutic agent that has withstood the test of time is mitomycin $\mathrm{C}$ (MMC) but evaluation of its clinical efficacy is difficult because so many investigators have used widely varying MMC doses, concentrations, instillation volumes and residence times, usually in heterogeneous patient populations. However, as early as 1993 Wientjes et al combined data from laboratory, animal and human studies with computer simulations to describe a compelling MMC regimen primarily based on optimizing diffusion down concentration gradients (Fick's first law). ${ }^{6}$ The same group followed up with

Accepted for publication April 11, 2003.

Study received institutional review board approval.

Supported by grants Progetti di Ricerca di Ateneo ex 60\% 19992000 and 2000-2001 from Tor Vergata University of Rome. Electromotive equipment provided by Physion Srl, Medolla, Italy.

* Corresponding author: Via Torrice n. 4, 00189 Rome, Italy (telephone: 390630311655; FAX: 390623188353; e-mail: sdistas@tin.it).

$\dagger$ Financial interest and/or other relationship with Physion Srl. a study showing the advantages of increased concentration gradients in animals and humans ${ }^{7}$ and then reported a phase III trial, in which the optimized regimen proved superior to a standard MMC regimen in patients with Ta grade I/II bladder cancers. ${ }^{8}$ However, results in subgroups with Tis, grade III and T1 disease were less definitive, although trends toward improvement were discernible. Therefore, it must be assumed that there are several reasons for the many failures that occur using intravesical MMC for high risk superficial bladder cancer. Under staging and/or incomplete disease resection are obvious causes applicable to all intravesical regimens. ${ }^{9}$ Another cause is the invasive depth of T1 tumors, which usually reaches beyond the required therapeutic concentrations of MMC no matter how optimal the intravesical treatment. ${ }^{6}$ Finally, aggressive high grade cancer cells are less chemosensitive, ${ }^{10}$ which may explain why Tis responds poorly to MMC. If Tis, grade III and $\mathrm{T} 1$ cancers require higher concentrations than can be delivered by passive transport, further acceleration of MMC administration rates with increased accumulation in tissues may improve the clinical response.

Electrokinetic forces accelerate drug delivery into and across biological membranes. MMC is nonionized within the tolerable physiological range and its electromotive mode of delivery is by electro-osmosis. Iontophoretic administration of ions ${ }^{11}$ is accompanied by an electrokinetic flow of water, which entrains solubilized MMC. ${ }^{12}$ Thus, the total flux of MMC becomes the sum of electromotive and passive transport rates, and correct selection of the total charge (current 
intensity $\times$ time) ensures that electromotive administration is the dominant and controllable transport mechanism. In the laboratory electromotive MMC increased delivery rates 4 to 7 -fold compared with passive transport. ${ }^{12}$

Therefore, although currently intravesical administration of chemotherapeutic drugs results in a high proportion of failures, electromotive acceleration of MMC would theoretically decrease the failure rate. We evaluated this concept in a multicenter, prospective, randomized study in patients with high risk superficial bladder cancer, comparing the efficacy of electromotive MMC with that of passive MMC in 2 patient groups and using BCG instillation in a third group as comparative treatment.

\section{PATIENTS AND METHODS}

Patient selection. Enrollment commenced in June 1994 and continued through March 2001. The patient population had histologically proven multifocal carcinoma in situ (Tis) of the bladder and most had concurrent p $\mathrm{T} 1$ papillary transitional cell carcinoma (table 1 ). All patients had adequate bone marrow reserve, normal renal function, normal liver function and a Karnofsky performance score of 50 to 100. All were assessed as reliable for long-term followup. Exclusion criteria included prior carcinoma of the bladder and/or upper urinary tract, other malignancies within 5 years of registration and pregnancy.

Study design. Figure 1 shows the study design. The patients supplied written informed consent to a document describing the investigational nature of the protocol.

Treatment schedules. Following initial evaluation patients underwent urinary cytology of the bladder and upper urinary tract, and multiple bladder biopsies. We performed complete transurethral resection (TUR) of all visible bladder tumor, ensuring that muscle was included in the resected specimens. Patients for whom the pathology report was equivocal or indicative of incomplete resection underwent re-staging TUR 4 to 5 weeks later.

Randomization and data collection were performed using a central computer. Patients were allocated to 1 of 3 treatment arms by blocked randomization across $8(2 \times 2 \times 2)$ strata resulting from 3 factors, namely Tis only vs Tis with concurrent $\mathrm{T} 1$ papillary tumors, grades III vs II concurrent T1 papillary tumors and multifocal vs unifocal concurrent T1 papillary tumors. This method ensured prognostic parity among the 3 treatment groups, that is $\mathrm{BCG}$, passive MMC and electromotive MMC (fig. 1). All groups were scheduled to receive an initial 6 intravesical treatments at weekly intervals commencing approximately 3 weeks after multiple biopsy/TUR procedures. Patients in the 3 groups who had a complete response to the initial 6 weekly treatments underwent a further 10 monthly instillations. If cancer persisted at 3 months, a second 6 -week course was given. If disease persisted at 6 months, there was a crossover to a 6 -week second line course of BCG for patients in the 2 MMC groups and electromotive MMC for patients in the BCG group (fig. 1).

Patients in the $2 \mathrm{MMC}$ arms were placed on fluid restriction and oral sodium bicarbonate before intravesical MMC treatments. ${ }^{6}$ Under ultrasound control the bladder was thoroughly drained by repositioning the catheter and/or patient. A dose of $40 \mathrm{mg}$ MMC with $960 \mathrm{mg}$ excipient $\mathrm{NaCl}$ dissolved in $100 \mathrm{ml}$ water was instilled and retained in the bladder for 60 minutes in the passive MMC arm and for 30 minutes with $20 \mathrm{~mA}$ pulsed electric current $(600 \mathrm{~mA} \cdot$ minute $)$ in the electromotive MMC arm. Patients in the BCG arm were treated intravesically with $81 \mathrm{mg}$ wet weight (mean \pm SEM $10.2 \pm$ $9.0 \times 10^{8} \mathrm{cfu}$ ) Pasteur BCG. Lyophilized BCG was suspended in $50 \mathrm{ml}$ bacteriostatic-free $\mathrm{NaCl} 0.9 \%$ solution. After draining the bladder the suspension was instilled and retained for 120 minutes.

Toxicity. Side effects were classified as local, systemic or allergic. Local toxicity was defined as culture proven bacterial cystitis, drug induced (chemical) cystitis and other localized effects. Systemic side effects were defined as fever exceeding $38 \mathrm{C}$, general malaise and fatigue, liver toxicity, BCG induced lung infection and BCG sepsis. Skin rash, arthralgia and arthritis were regarded as allergic reactions. The severity of side effects was classified by the treating physician as requiring no delay, delay or indefinite postponement of instillations.

Patient followup. Response to treatment was assessed with cystoscopy, biopsy and urinary cytology. Patients underwent bladder biopsies at 3 and 6 months, and thereafter only if indicated by suspicious cytological findings or cystoscopy. In tumor-free cases cystoscopy and urinary cytology were repeated at 3-month intervals for 2 years, 6-month intervals for 3 years and yearly thereafter.

Patient evaluation. Complete response was defined as histological disappearance of malignancy on bladder biopsy and resolution of abnormal cytological findings. Time to the first recurrence (disease-free interval) was defined as the time from randomization to the first cystoscopy at which recurrence was observed. Time to progression was defined as the time from randomization to the first appearance of muscle invasive disease (stage T2 or greater) or to a change in treatment strategy, implying impending progression (cystectomy for persistent Tis). Survival was defined as the time from randomization to the date of death from any cause. Patients without recurrence or progression were censored at the last followup cystoscopy. Patients lost to followup were censored at the last known day of survival.

MMC pharmacological studies. A total of 15 patients in the passive and electromotive MMC arms, respectively, underwent pharmacological evaluation during the first intravesical instillation. Blood samples $(10 \mathrm{ml})$ were drawn before and at certain time points after MMC instillation, including 0, 5, $15,30,45,60,90,120,180,300$ and 480 minutes. The plasma

TABLe 1. Patient characteristics

\begin{tabular}{|c|c|c|c|c|c|c|c|}
\hline & \multicolumn{2}{|r|}{ BCG } & & $\begin{array}{l}\text { Passive } \\
\text { MMC }\end{array}$ & \multicolumn{2}{|c|}{$\begin{array}{c}\text { Electromotive } \\
\text { MMC }\end{array}$} & $\mathrm{p}$ Value \\
\hline \multicolumn{8}{|l|}{ Demographics: } \\
\hline Median age (IQR) & \multicolumn{2}{|c|}{$66.5(13)$} & \multicolumn{2}{|c|}{$68.5(16.5)$} & \multicolumn{2}{|c|}{$64.5(18.5)$} & 0.28 (Kruskal-Wallis \\
\hline No. men/women (\%) & 27 & $(75.0) / 9(25.0)$ & 26 & $(72.2) / 10(27.8)$ & 26 & $(72.2) / 10(27.8)$ & 1.00 (Fisher exact test) \\
\hline \multicolumn{8}{|l|}{ No. stratified randomization (\%): } \\
\hline Ca in situ only & 3 & $(8.3)$ & 3 & $(8.3)$ & 4 & $(11.1)$ & 1.00 (Fisher exact test) \\
\hline $\mathrm{Ca}$ in situ + concurrent papillary p $\mathrm{T} 1$ & 33 & $(91.7)$ & 33 & $(91.7)$ & 32 & $(88.9)$ & \\
\hline Grade $2 \mathrm{Ca}$ in situ + concurrent papillary pT1 & 19 & $(57.6)$ & 19 & $(57.6)$ & 18 & $(56.3)$ & 1.00 (Fisher exact test) \\
\hline Grade $3 \mathrm{Ca}$ in situ + concurrent papillary pT1 & 14 & $(42.4)$ & 14 & $(42.4)$ & 14 & $(43.7)$ & \\
\hline Unifocal Ca in situ + concurrent papillary pT1 & 12 & $(36.4)$ & 10 & $(30.3)$ & 12 & $(37.5)$ & 0.90 (Fisher exact test) \\
\hline Multifocal $\mathrm{Ca}$ in situ + concurrent papillary $\mathrm{pT} 1$ & 21 & $(63.6)$ & 23 & $(69.7)$ & 20 & $(62.5)$ & \\
\hline Median mos followup (IQR) & 43 & $(23.5)$ & 42 & $(23)$ & 45 & $(23.5)$ & $\begin{array}{l}0.94 \text { (Kruskal-Wallis } \\
\text { nonparametric test) }\end{array}$ \\
\hline
\end{tabular}




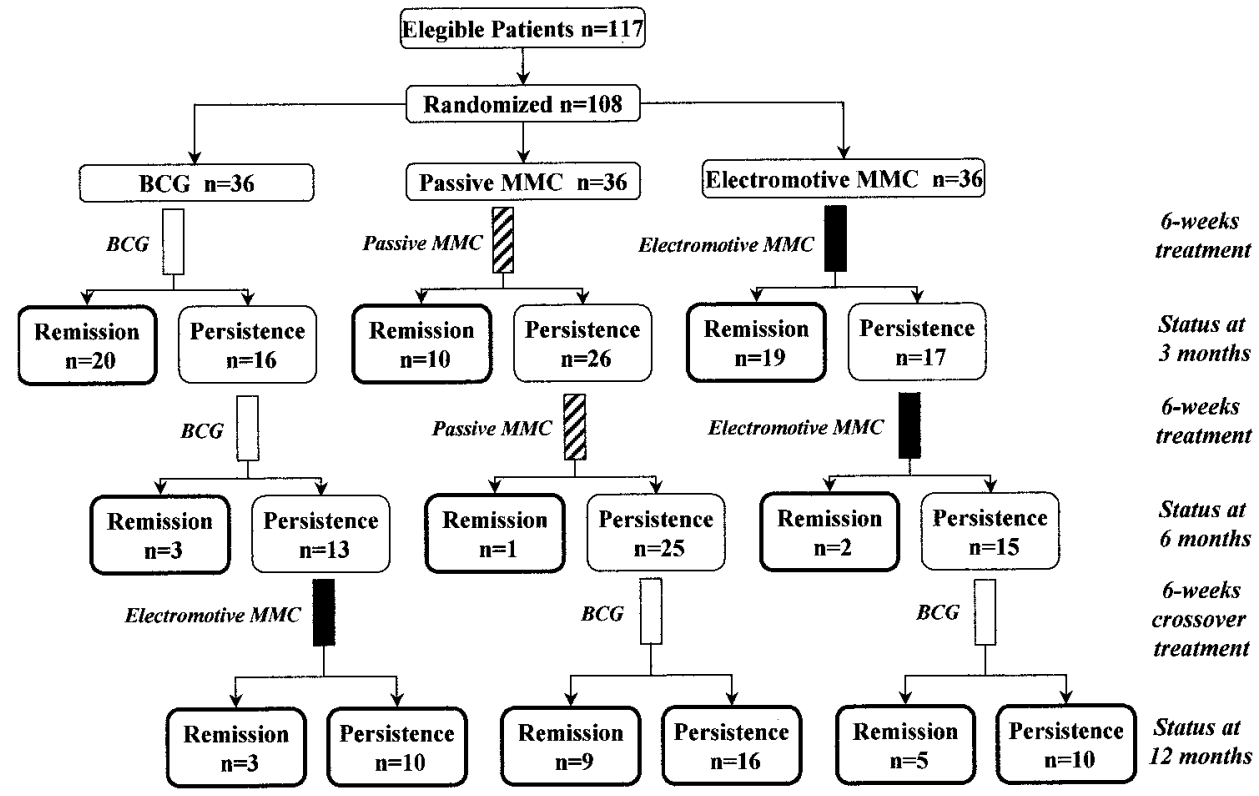

FIG. 1. Trial flow diagram incorporating patient numbers and status to 12 months

fraction was stored at $-70 \mathrm{C}$ until analysis. ${ }^{12}$ During intravesical passive and electromotive MMC bladder content samples were obtained at 5, 15, 30, 45 and 60, and 5, 15 and 30 minutes, respectively, and stored at $-70 \mathrm{C}$ until analysis. After the instillation period (60 and 30 minutes) the solution was drained and volume was measured. Bladder volume at sampling times (Vt) included the original $100 \mathrm{ml}$ instillation (Vo) plus urine. Assuming a constant rate of urine inflow, the measured $\mathrm{MMC}$ concentration $(\mathrm{Cm})$ was normalized to its concentration $(\mathrm{Ct})$ in the original $100 \mathrm{ml}$ volume using the formula, $\mathrm{Ct}=\mathrm{Cm} \times \mathrm{Vt} / \mathrm{Vo}$.

Statistical methods. All clinical analyses were performed on an intent to treat basis. The primary study end point was a complete response 3 and 6 months following treatment, and the aim was to evaluate differences between responses to passive MMC, estimated at $33 \%,{ }^{13}$ and to electromotive MMC, anticipated to be equivalent to that of a BCG response rate of $70 \% .^{14}$ For a power of 0.8 and an $\alpha$ level of 0.05 (2-sided) we calculated a sample size of 108 patients, that is 3 groups of 36 patients each. The nonparametric Fisher exact test was applied to tables and the Kruskal-Wallis test was used for multiple median comparisons. Time to recurrence, time to progression and overall survival were studied by the Kaplan-Meier method and comparisons were made by the log rank test. All tests were 2 -sided. Resulting $p$ values are reported and significance was assumed at the 0.05 level. Pharmacokinetics data are expressed as the mean \pm 1 SEM of 15 observations per group. Kinetic parameters (elimina- tion half-life and constants, exponential decay and regression analyses) were calculated using a computer program. Differences were considered significant at $\mathrm{p}<0.05$.

\section{RESULTS}

Patient characteristics. Re-staging TUR was considered necessary in 28 of 117 patients following initial TUR and biopsies. In 5 of these patients residual superficial tumor was resected, while 3 were under staged (pT2) and, therefore, ineligible. Another 3 patients refused, 2 had problems with medications and 1 had a cerebrovascular accident prior to instillation. The remaining 108 patients who had diffuse Tis, including 98 in whom associated papillary T1 tumors were resected, were randomized and commenced intravesical treatments. Table 1 lists demographic and descriptive statistics on the patients. Pertinent characteristics in the 3 treatment arms were comparable.

Toxicity (table 2). Local side effects and systemic side effects were significantly more prominent in the BCG arm than in the 2 MMC arms. There were no statistical differences between the 2 MMC arms, although there was a trend toward increasing numbers and side effect severity in the electromotive MMC group. There were significant differences in modified treatment between the BCG and MMC arms. A total of 32 patients on BCG, 34 on passive MMC and 33 on electromotive MMC completed treatment. Nine patients $(8.3 \%)$ did not complete the first 6 weekly treatments due to

TABLE 2. Adverse effects in patients who received at least 1 or more intravesical treatments

\begin{tabular}{|c|c|c|c|c|}
\hline Adverse Effect & No. BCG (\%) & No. Passive MMC (\%) & No. Electromotive MMC (\%) & $\begin{array}{l}\mathrm{p} \text { Value (Fisher } \\
\text { exact test) }\end{array}$ \\
\hline Urinary frequency & $21(58.3)$ & $6(16.7)$ & 7 (19.4) & 0.001 \\
\hline Bacterial cystitis & $9(25.0)$ & $7(19.4)$ & 7 (19.4) & 0.874 \\
\hline Drug induced cystitis & $24(66.7)$ & $9(25.0)$ & $13(36.1)$ & 0.001 \\
\hline Visible hematuria & $26(72.2)$ & $6(16.7)$ & $8(22.2)$ & 0.001 \\
\hline Prostatitis & $1(2.8)$ & 0 & 0 & 1.000 \\
\hline Epididymitis & $1 \quad(2.8)$ & 0 & 0 & 1.000 \\
\hline Fever & $7(19.4)$ & 0 & 0 & 0.001 \\
\hline General malaise & $11(30.5)$ & $1(2.8)$ & 0 & 0.001 \\
\hline Fatigue & $16(44.4)$ & 0 & $1 \quad(2.8)$ & 0.001 \\
\hline Allergic reactions & 0 & $2 \quad(5.6)$ & $3 \quad(8.3)$ & 1.000 \\
\hline \multicolumn{5}{|l|}{ Treatment modified: } \\
\hline No & $10(27.8)$ & $26(72.2)$ & $21(58.4)$ & \\
\hline Yes, continued & $22(61.1)$ & $8(22.2)$ & $12(33.3)$ & 0.003 \\
\hline Yes, stopped & $4(11.1)$ & $2(5.6)$ & $3(8.3)$ & \\
\hline
\end{tabular}


severe toxicity, including 4 in the BCG arm due to prostatitis, persistent gross hematuria, drug induced cystitis and systemic side effects, 2 in the passive MMC arm and 3 in the electromotive MMC arm due to allergic reactions. No hematological toxicity was observed, no patients had a contracted bladder and no life threatening adverse effects or treatment related deaths occurred.

Patient evaluation and followup (fig. 1, table 3). The response rate at 3 months for the passive MMC, electromotive MMC and BCG groups was 10 of 36, 19 of 36 and 20 of 36 patients, respectively. At 6 months the corresponding rates were 11 of 36, 21 of 36 and 23 of 36 patients. At each time point the electromotive MMC response rate was significantly superior to that of passive MMC.

A total of 53 patients underwent crossover treatments, including 40 with persistent disease who changed from passive MMC (25) and electromotive MMC (15) to a 6-week course of BCG, while 13 in whom BCG failed changed to a course of electromotive MMC (fig. 1). Of these patients 17 $(32 \%)$ had a complete response (fig. 1, table 3). Figure 1 shows the status of all patients at 12 months. Of the 108 patients $72(67 \%)$ had a complete response, including 26 of 36 (72\%) in the original BCG arm, 20 of 36 (56\%) in the original passive MMC arm and 26 of $36(72 \%)$ in the original electromotive MMC arm. The difference was not significant.

Recurrence. During a median followup of 43 months there was recurrence in 65 of the 108 patients, that is a total of 126 episodes, including 37 in the BCG and electromotive MMC groups, and 54 in the passive MMC group (table 3). There were 2 or more recurrences in 38 patients $(35 \%)$. A total of 43 patients (49\%) remained free of recurrence, including 17 in the BCG arm, 9 in the passive MMC arm and 17 in the electromotive MMC arm. Figure 2 shows Kaplan-Meier curves for the disease-free interval. Median time to first recurrence was 35 months for the electromotive MMC group, which was significantly longer than 19.5 months for the passive MMC group and 26 months for the BCG group (table 3).

Progression. Figure 2 shows Kaplan-Meier curves for time to disease progression and an overall log rank test simultaneously comparing the 3 treatment groups. Five patients underwent cystectomy for persistent Tis at 9 to 12 months and 15 progressed to muscle invasive disease with subsequent cystectomy (table 3 ). Ten of the 15 patients first showed progression at 24 to 39 months, while in 5 progression was detected at 12 to 24 months.

Survival. Figure 2 shows Kaplan-Meier plots of time to death from any cause and from bladder cancer. There was an overall mortality of 32 of 108 cases $(29.6 \%)$, including 11 in the BCG, 12 in the passive MMC and 9 in the electromotive MMC groups (table 3 ). Of the 20 patients treated with cystectomy 19 died of disseminated bladder cancer, of whom 16 had failed to respond to each 6-week course of intravesical therapy and subsequent crossover (table 3).
MMC pharmacokinetics. Figure 3 shows plasma MMC following intravesical passive transport and intravesical electromotive administration. During passive transport peak plasma concentrations $(7.8 \pm 1.7 \mathrm{ng} / \mathrm{ml})$ were attained at 60 minutes. Levels then decayed by first order kinetics with an estimated half-life of 97 minutes and $\beta=(2.59 \pm 0.17) \times$ $10^{-3}$. During electromotive administration plasma MMC at all time points was significantly higher than after passive transport. Peak plasma concentrations $(42.9 \pm 7.1 \mathrm{ng} / \mathrm{ml})$ were achieved within 30 minutes and the subsequent elimination phase followed first order kinetics with $\beta=(2.79 \pm$ $0.24) \times 10^{-3}$ and an estimated half-life of 56 minutes.

Figure 4 shows the decay of MMC concentrations with passive and electromotive transport with the corrective factor $\mathrm{Ct}=\mathrm{Cm} \times \mathrm{Vt} / \mathrm{Vo}$ applied to all MMC measurements in intravesical samples. The best fit for passive transport $(r=0.9998)$ was the 1-phase exponential decay equation, $\mathrm{y}=199^{-0.275 \mathrm{x}}+200.8$, with an estimated disappearance half-life of 2.52 minutes, showing that the absorption process was almost complete within 15 minutes and at 60 minutes approximately $50 \%$ of the initial MMC content was absorbed. The decay of MMC concentrations with electromotive administration also showed a best fit 1-phase exponential decay equation of $\mathrm{y}=325.7^{-0.321 \mathrm{x}}+74.2(\mathrm{r}=0.9999)$ with an estimated disappearance half-life of 2.16 minutes and at 30 minutes more than $80 \%$ of the initial MMC content absorbed.

\section{DISCUSSION}

In 1994 the available literature provided a wide variety of Tis response rates to MMC and eventually we selected the $33 \%$ rate supplied in a succinct editorial comment. ${ }^{13}$ Equating electromotive MMC with BCG (70\%) was partly supposition and partly based on preliminary laboratory studies. ${ }^{12}$ It is an interesting observation that in the current study almost all MMC uptake for each administration technique was complete after about 15 minutes of residence time. This cutoff interval was also observed for tissue MMC measurements in the laboratory and it was attributed at that time to MMC degradation and metabolism balancing continuing uptake, ${ }^{12}$ an hypothesis that obviously is no longer tenable.

There is a rationale for the particular MMC solution (40 $\mathrm{mg} / 100 \mathrm{ml}$ water) used in the current study. Intravesical electromotive administration of any drug requires volumes of $100 \mathrm{ml}$ or more ${ }^{15}$ to ensure even drug distribution throughout the bladder wall. We initially selected $100 \mathrm{ml}$ volumes for patients in the passive transport group because it matched that in the electromotive group. However, we have since become increasingly aware of important differences in MMC formulations. The formulation used in this study and throughout Europe contains $\mathrm{NaCl}$ as an excipient with an MMC-to- $\mathrm{NaCl}$ ratio of $1: 24 \mathrm{mg}$, so that routine intravesical solutions are markedly hypertonic. Passive transport is the algebraic sum of diffusive flux and osmotic solvent drag, ${ }^{16}$ a

TABLE 3. Treatment efficacy based on intent to treat analysis

\begin{tabular}{|c|c|c|c|c|}
\hline & BCG & Passive MMC & Electromotive MMC & $\mathrm{p}$ Value \\
\hline \multicolumn{5}{|l|}{$\%$ Complete response $(95 \% \mathrm{CI}):$} \\
\hline $6 \mathrm{Mos}$ & $63.9(46.2-79.2)$ & $30.5(16.3-48.1)$ & $58.3(40.7-74.4)$ & 0.012 (Fisher exact test) \\
\hline Crossover & $35.0(20.6-51.7)$ & & $23.1(5.0-53.8)$ & 0.511 (Fisher exact test) \\
\hline$\%$ Pts $(95 \%$ CI $)$ & $52.8(35.5-69.6)$ & $75.0(57.8-87.9)$ & $52.8(35.5-69.6)$ & 0.092 (Fisher exact test) \\
\hline Median mos to recurrence $(95 \% \mathrm{CI})$ & $26 \quad(21-37)$ & $19.5(12-27)$ & $35 \quad(21-46)$ & 0.013 (Fisher exact test) \\
\hline$\%$ Progression $(95 \% \mathrm{CI})$ & $16.7 \quad(6.4-32.8)$ & $22.2(10.1-39.1)$ & $16.7(6.4-32.8)$ & 0.861 (Fisher exact test) \\
\hline \multicolumn{5}{|l|}{$\begin{array}{l}\% 5 \text {-Yr estimated survival probability } \\
(95 \% \mathrm{CI}) \text { : }\end{array}$} \\
\hline Bladder $\mathrm{Ca}$ & $78.0(54.1-90.4)$ & $75.3(89.6-88.3)$ & $78.5(57.9-89.8)$ & $\begin{array}{l}0.943 \text { (likelihood ratio } \\
\text { homogeneity test sta- } \\
\text { tistic) }\end{array}$ \\
\hline
\end{tabular}



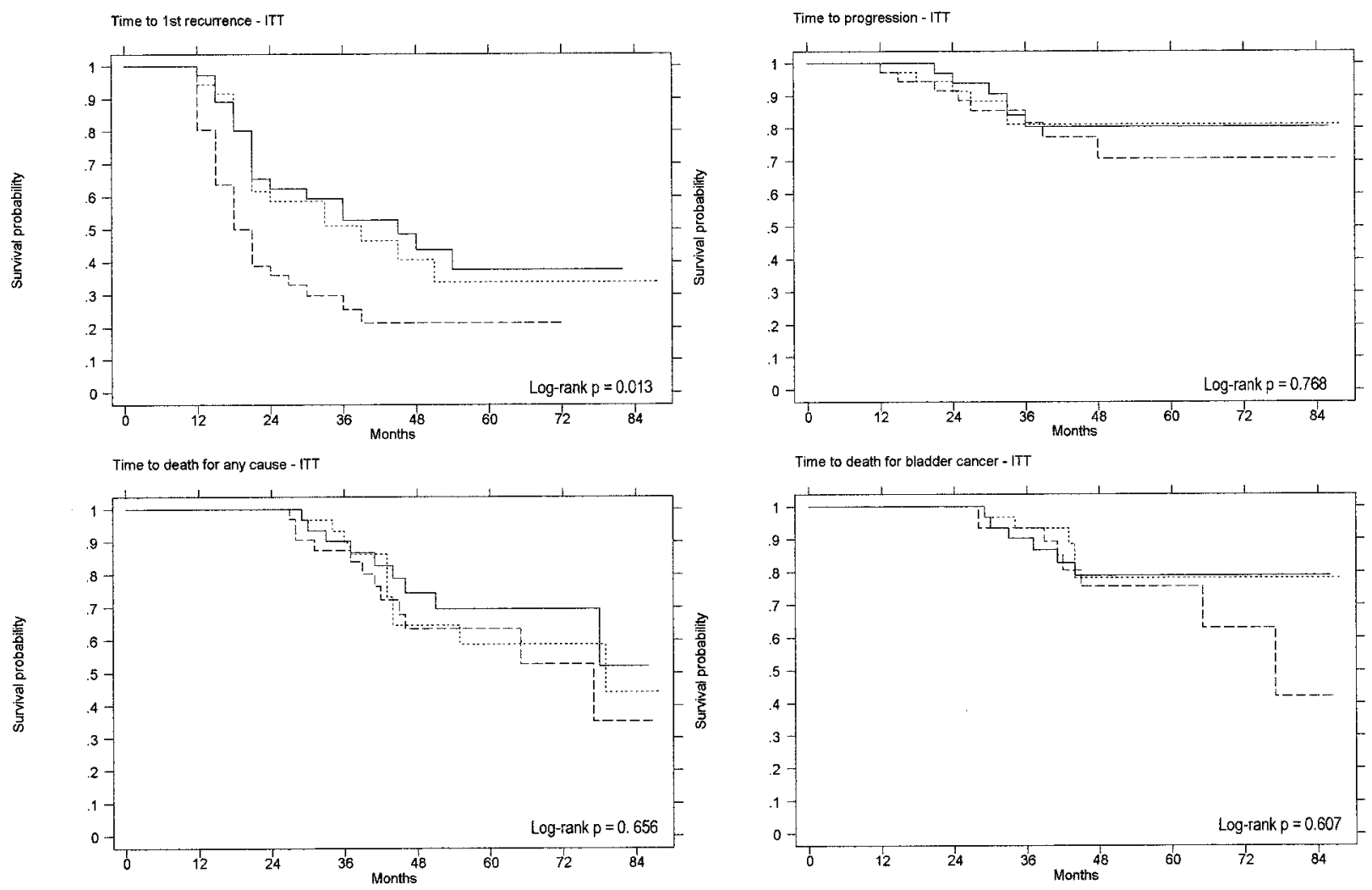

FIG. 2. Kaplan-Meier curves and log rank tests for intent to treat (ITT) data on time to recurrence, to progression, to death from any cause and to death from bladder cancer. Dotted line represents BCG. Dashed line represents passive MMC. Solid line represents electromotive MMC.

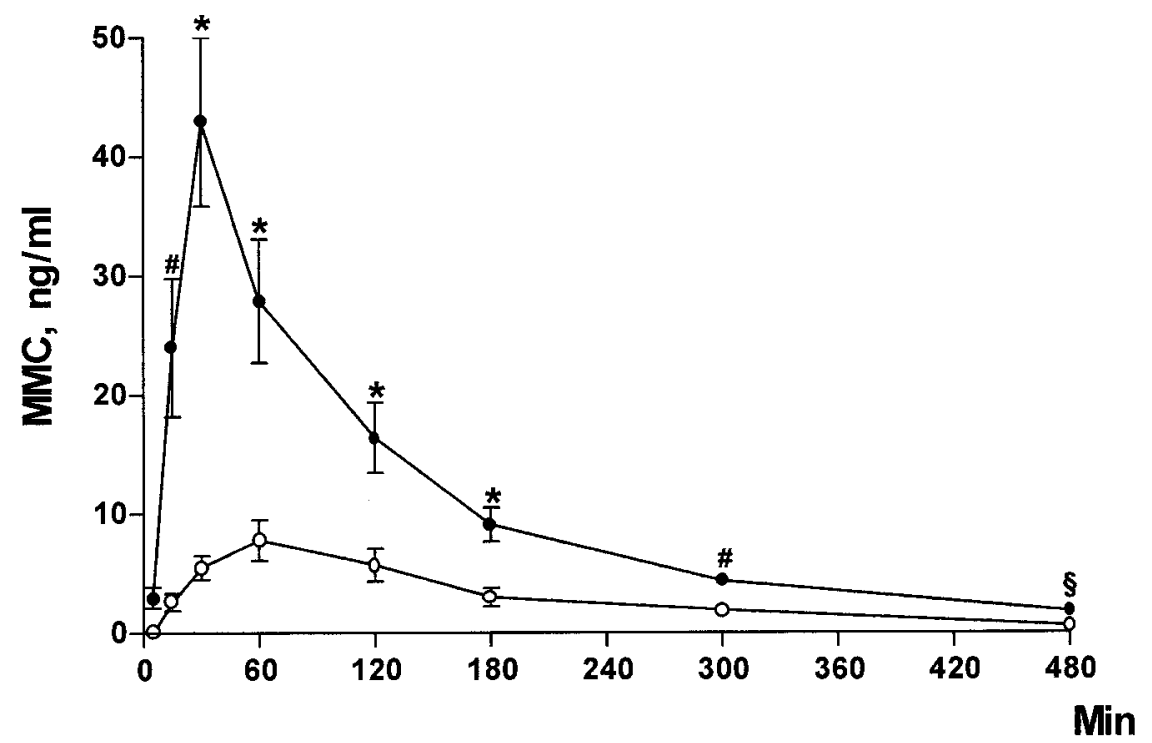

Fig. 3. Mean plasma MMC \pm 1 SEM of 15 measurements per time point after intravesical administration of $40 \mathrm{mg}$ MMC in $100 \mathrm{ml}$ in passive transport (open circles) and electromotive administration (filled circles) groups. \#, vs passive diffusion $p<0.01$. *, vs passive diffusion $\mathrm{p}<0.001 . \S$, vs passive diffusion $\mathrm{p}<0.05$

phenomenon that is judiciously applied for peritoneal dialysis $^{17}$ and which has also been demonstrated in carcinoma cells. ${ }^{18}$ Increasing the concentration increases osmotic transport away from tissues into drug solutions and offsets the increased diffusion from drug solutions into tissues. Thus, there is no linear relationship of transport rate-to-drug concentration, as demonstrated by Gao et al using hypotonic (27 mOsm or less) MMC/mannitol formulations. ${ }^{7}$

Although electromotive administration markedly in- creased MMC transport, plasma levels in the current study remained well below toxic concentrations $(400 \mathrm{ng} / \mathrm{ml})$. With 1 or 2, 6 weekly schedules followed by 10 monthly instillations all 3 treatment modes were prolonged and adverse effects were plentiful, although significantly more so with BCG. Nevertheless, only 9 of the 108 patients failed to complete the first 6 -week schedule. Although side effects consumed substantial medical resources, there were no life threatening events and no permanent bladder contracture. 


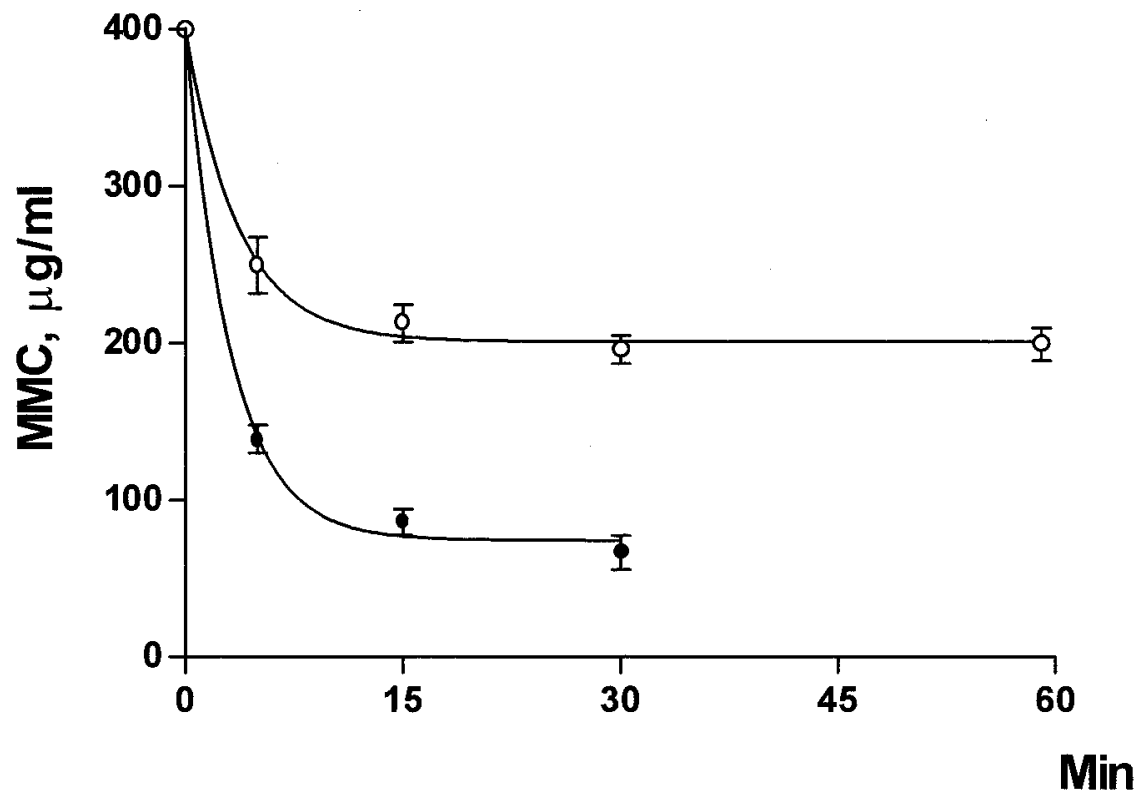

Fig. 4. Mean decay of initial $40 \mathrm{mg}$ intravesical MMC in $100 \mathrm{ml} \pm 1 \mathrm{SEM}$ of 15 measurements per time point during passive transport for 60 minutes (open circles) and electromotive administration for 30 minutes (filled circles).

Clinical response rates at 3 and 6 months validated the prediction that electromotive enhancement of MMC delivery into high risk superficial bladder cancers would provide results superior to those achieved using passive MMC. Notably the crossover to BCG and electromotive MMC at 6 months further increased the number of responders. Nevertheless, the complete response rate and time to recurrence are an incomplete guide to the eventual fate of these patients at high risk. ${ }^{19}$ At a median followup of 43 months 20 of the 108 patients $(19 \%)$ underwent cystectomy and 19 died of disseminated bladder cancer. With hindsight 2 modifications to the study may have improved this situation. 1) Followup cystoscopy 4 to 5 weeks after initial evaluation in all patients would have detected under staging in a few and probable incomplete resection of $\mathrm{T} 1$ tumors in several more. ${ }^{20} 2$ ) Of the patients $80 \%$ in whom metastatic disease developed had failed to respond to the 2, 6-week courses of intravesical therapy and subsequent crossover, indicating that these particular superficial cancers were aggressively malignant. Prompt cystectomy should have been urged forcefully rather than provided as an option to continued surveillance.

\section{CONCLUSIONS}

Intravesical electromotive MMC provides a superior response rate in patients with high risk superficial bladder cancer compared with passive MMC transport. The indications are that these improved response rates match those induced by BCG, so that electromotive MMC may be an alternative or complementary therapy. There remains an unresolved theoretical concern. At least in animals MMC is carcinogenic and could expose patients to double jeopardy, that is the original cancerous diathesis plus MMC induced cancerous diathesis. All of our patients are subject to lifelong surveillance, which should assist in resolving this issue.

\section{REFERENCES}

1. Jakse, G., Loidl, W., Seeber, G., and Hofstädter, F.: Stage T1, grade 3 transitional cell carcinoma of the bladder: an unfavorable tumor? J Urol, 137: 39, 1987

2. Abel, P. D.: Follow-up of patients with "superficial" transitional cell carcinoma of the bladder: the case for a change in policy. Br J Urol, 72: 135, 1993

3. Bostwick, D. G.: Natural history of early bladder cancer. J Cell Biochem, suppl., 16I: 31, 1992
4. Cookson, M. S., Herr, H. W., Zhang, Z.-F., Soloway, S., Sogani, P. C. and Fair, W. R.: The treated natural history of high risk superficial bladder cancer: 15-year outcome. J Urol, 158: 62, 1997

5. Kim, J. C. and Steinberg, G. D.: The limits of bacillus CalmetteGuérin for carcinoma in situ of the bladder. J Urol, 165: 745, 2001

6. Wientjes, M. G., Badalament, R. A. and Au, J. L.: Use of pharmacologic data and computer simulations to design an efficacy trial of intravesical mitomycin $C$ therapy for superficial bladder cancer. Cancer Chemother Pharmacol, 32: 255, 1993

7. Gao, X., Au, J. L., Badalament, R. A. and Wientjes, M. G: Bladder tissue uptake of mitomycin $\mathrm{C}$ during intravesical therapy is linear with drug concentration in urine. Clin Cancer Res, 4: 139,1998

8. Au, J. L., Badalament, R. A., Wientjes, M. G., Young, D. C., Warner, J. A., Venema, P. L. et al: Methods to improve efficacy of intravesical mitomycin C: results of a randomized phase III trial. J Natl Cancer Inst, 93: 597, 2001

9. Herr, H. W.: The value of a second transurethral resection in evaluating patients with bladder tumors. J Urol, 162: 74, 1999

10. Schmittgen, T. D., Wientjes, M. G., Badalament, R. A. and Au, J. L.: Pharmacodynamics of mitomycin $\mathrm{C}$ in cultured human bladder tumors. Cancer Res, 51: 3849, 1991

11. Phipps, J. B., Padmanabhan, R. V. and Lattin, G. A.: Iontophoretic delivery of model inorganic and drug ions. J Pharm Sci, 78: 365, 1989

12. Di Stasi, S. M., Giannantoni, A., Massoud, R., Dolci, S., Navarra, P., Vespasiani, G. et al: Electromotive versus passive diffusion of mitomycin $\mathrm{C}$ into human bladder wall: concentration-depth profiles studies. Cancer Res, 59: 4912, 1999

13. Soloway, M. S.: Editorial comment. J Urol, 143: 36, 1990

14. Lamm, D. L.: Carcinoma in situ. Urol Clin North Am, 19: 499, 1992

15. Lugnani, F., Mazza, G., Cerulli, N., Rossi, C. and Stephen, R. L.: Iontophoresis of drugs in the bladder wall: equipment and preliminary studies. Artif Org, 17: 8, 1993

16. Katchalsky, A. and Curran, P. F.: Nonequilibrium Thermodynamics in Biophysics. Cambridge: Harvard University Press, 1965

17. Henderson, L. W.: Peritoneal ultrafiltration dialysis: enhanced urea transfer using hypertonic peritoneal dialysis fluid. J Clin Invest, 45: 950, 1966

18. Stephen, R. L., Novak, J. M., Jensen, E. M., Kablitz, C. and Buys, S. S.: Effect of osmotic pressure on uptake of chemotherapeutic agents by carcinoma cells. Cancer Res, 50: 4704, 1990

19. Montie, J. E.: Intravesical therapy for bladder cancer: empiricism at the helm. J Natl Cancer Inst, 93: 572, 2001

20. Herr, H. W. and Reuter, V. E.: Progression of T1 bladder tumors: better staging or better biology? Cancer, 86: 908, 1999 